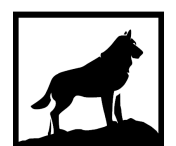

Michigan

Technological

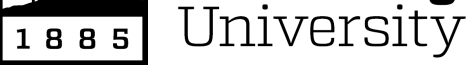

Michigan Technological University

Digital Commons @ Michigan Tech

2017

PERFORMANCE SIMULATION AND OPTIMIZATION OF A

SIMULTANEOUS TRANSMIT AND RECEIVE PHASED ANTENNA

ARRAY USING ADAPTIVE BEAMFORMING AND GENETIC ALGORITHM TECHNIQUES

Ian Thomas Cummings

Michigan Technological University, itcummin@mtu.edu

Copyright 2017 lan Thomas Cummings

Recommended Citation

Cummings, lan Thomas, "PERFORMANCE SIMULATION AND OPTIMIZATION OF A SIMULTANEOUS

TRANSMIT AND RECEIVE PHASED ANTENNA ARRAY USING ADAPTIVE BEAMFORMING AND GENETIC ALGORITHM TECHNIQUES", Open Access Master's Report, Michigan Technological University, 2017.

https://doi.org/10.37099/mtu.dc.etdr/326

Follow this and additional works at: https://digitalcommons.mtu.edu/etdr

Part of the Signal Processing Commons 


\title{
PERFORMANCE SIMULATION AND OPTIMIZATION OF A SIMULTANEOUS TRANSMIT AND RECEIVE PHASED ANTENNA ARRAY USING ADAPTIVE BEAMFORMING AND GENETIC ALGORITHM TECHNIQUES
}

By

Ian Thomas Cummings

\begin{abstract}
A REPORT
Submitted in partial fulfillment of the requirements for the degree of MASTER OF SCIENCE

In Computer Engineering
\end{abstract}

MICHIGAN TECHNOLOGICAL UNIVERSITY

2017

(C)2017 Ian Thomas Cummings 
This report has been approved in partial fulfillment of the requirements for the Degree of MASTER OF SCIENCE in Computer Engineering.

Department of Electrical and Computer Engineering

\author{
Report Co-Advisor: Dr. Timothy C. Havens \\ Report Co-Advisor: Dr. Timothy Schulz \\ Committee Member: Dr. Michael Roggemann \\ Department Chair: Dr. Daniel Fuhrmann
}


To my family, and the many mentors who helped me make it this far. 


\section{Contents}

$\begin{array}{lll}1 & \text { Introduction } & 7\end{array}$

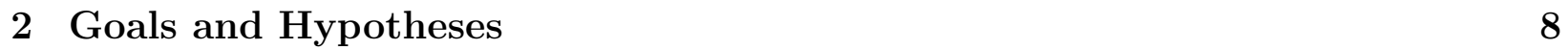

3 Methods $r$

3.1 Array Configuration \& Input Signal . . . . . . . . . . . . . . . . . 8

3.2 Transmit \& Receive Beamformer Optimization . . . . . . . . . . . . . 9

3.3 Transmit \& Receive Coupling Model . . . . . . . . . . . . . . . . . . . . . . 10

3.4 Signal Transmission \& Reflection Model . . . . . . . . . . . . . . . . . . . . 10

3.5 Reference Signal Model . . . . . . . . . . . . . . . . . . . . 12

3.6 Self-Interference Cancellation . . . . . . . . . . . . . . . . . 12

3.7 Array Performance Metric \& Optimization . . . . . . . . . . . . . . . . . 12

4 Results \& Discussion $\quad 13$

4.1 Array Partitions \& Beam Patterns . . . . . . . . . . . . . . . . . . 13

4.2 Array Performance Metric Summary . . . . . . . . . . . . . . . 18

4.3 Realized Self-Interference Magnitude . . . . . . . . . . . . . . . 19

5 Conclusions $\quad 21$

5.1 Future Work . . . . . . . . . . . . . . . . . . . . . . 22 


\section{Acknowledgements}

This material is based upon work supported by the National Science Foundation Graduate Research Fellowship Program under Grant No. 1546592. Any opinions, findings, and conclusions or recommendations expressed in this material are those of the author(s) and do not necessarily reflect the views of the National Science Foundation.

I would like to thank my Co-Advisors, Dr. Timothy Havens and Dr. Timothy Schulz, for their personal investment in my learning, growth, and advancement.

I would also like to thank Dr. Jon Doane of MIT Lincoln Laboratory RF Technology Group for reaching out to provide this amazing research topic, providing a great deal of feedback and guidance during the project, and for furnishing us with a realistic S-parameter matrix for our antenna array.

Finally, I would like to thank Dr. Adam J. Wachtor, Dr. Eric B. Flynn, and Dr. Chuck Farrar of Los Alamos National Laboratory, and Kevin Gregory of Electro-Optics Technology, Inc. for their encouragement and support in my pursuit of graduate studies. 


\section{Abstract}

The development of simultaneous transmit and receive capabilities is on the cutting-edge of research in phased array technology $[1,2,3]$. The large disparity in power between the transmitted and received signals in antenna systems has traditionally prevented operation in a simultaneous mode. However, simultaneous transmit and receive offers great opportunities for increased capabilities and performance in communications, radar, and electronic warfare applications [3]. This technology will be made feasible by realizing a high level of isolation between the transmitted and received signals through a variety of techniques. This work explores the feasibility of choosing non-standard array partitions that-when paired with the appropriate beamforming techniques - significantly reduce the self-interference between transmit and receive channels. 


\section{Introduction}

Phased arrays of antennas leverage relative phase between antennas to implement complex spatial filtering for transmitted and received signals. The relative phase information across antennas can be used to direct transmitted signals or listen for signals along a specified look angle. Additionally, depending on the number of antennas present, the array can be configured to avoid transmitting to or receiving from a given direction. The process of specifying these look angles is called beamforming. Historically, however, these systems have been limited to choosing between transmitting and receiving at any given moment. This limitation arises from the coupling between transmitting and receiving elements and the vastly disparate power levels involved in transmit and receive operation.

Efforts originated at Massachusetts Institute of Technology Lincoln Laboratories (MITLL) in the RF Technology Group to enable simultaneous transmit and receive operations in an antenna array through sophisticated beamforming and filtering techniques [1]. Specifically, array hardware was developed such that any antenna could be configured as either a transmit or receive element, and the receive hardware acted as a reference measurement channel when the antenna was in a transmitting mode [1]. The work derived the optimal transmit and receive beamformers and self-interference cancellation filter to maximize transmit and receive isolation in an array intended for communication operations [1]. Furthermore, it outlined a metric to characterize the total performance of these beamforming and filtering techniques [1]. Finally it demonstrated the success of these techniques by modeling a representative array in Ansys HFSS [1].

This technology is referred to as aperture-level simultaneous transmit and receive (ALSTAR) [1], and it will enable multi-function operation of reconfigurable antenna arrays [3]. This will open up the possibility for radar, communication, and electronic warfare capabilities in a single array. In order for such a system to be realized, a model of these functions and their interactions must be developed. Such a model must allow for optimization over the configuration parameter space, including which antennas are used to transmit and receive. This work describes the progress towards a quasi-narrowband model of the array presented in [1] operated in a radar mode.

In the standalone radar case, ALSTAR functionality becomes most relevant when the transmitter is constrained to operate at low power or the target presents a detrimentally attenuated return (due to extreme range, abnormal path attenuation, or incredibly small radar cross section, RCS). In such situations, the radar could increase the length of the transmitted pulse in order to increase the energy received at the target and reflected back to the array. However, in traditional systems, the length of this pulse is constrained to the round-trip time from the array to the target and back. The ability to isolate target returns at the receive antennas while transmitting would remove this constraint on pulse length. 


\section{Goals and Hypotheses}

We hypothesized that the partitioning of an array into transmit and receive antennas could have a significant affect on the achievable transmit-receive isolation for that array. We also hypothesized that a non-traditional partition could potentially outperform standard partitions - i.e., splitting the array into equal contiguous regions.

In order to explore these hypotheses, we developed a model that could accept array configuration parameters and use them to simulate the behavior of the array in a radar mode. It also calculated a performance metric for that configuration of the array. That model was then fed into a suitable optimization algorithm to select the array partition and beamformers that led to the highest performance.

\section{Methods}

\subsection{Array Configuration \& Input Signal}

The following simulated experiment investigated the feasibility and effectiveness of such a system. This work focused on simulating the $5 \times 10$ element rectangular antenna array described in detail in $[1,2]$. The transmitted pulse used in this work, denoted as $s_{i}(t)$, was a swept-sine with a center frequency of $2.45 \mathrm{GHz}$ and bandwidth of $100 \mathrm{M} \mathrm{Hz}$, comparable to the transmitted signal in [1]. Total transmitted power was set at $30 \mathrm{dBm}$. The simulation was conducted in complex baseband with a sample rate of $100 \mathrm{MHz}[4,5]$. The pulseshown in Figure 1-was long enough that a significant portion of the pulse remained to be transmitted when the leading edge of the reflection arrived back at the array. 

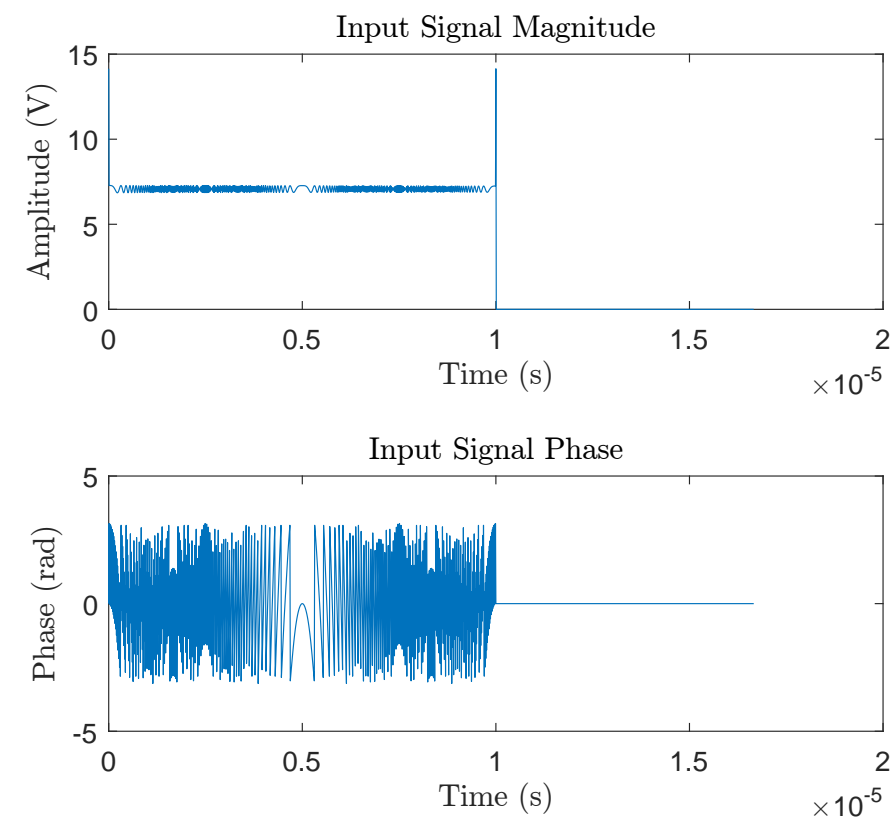

Figure 1: The radar pulse to be transmitted by the array.

\subsection{Transmit \& Receive Beamformer Optimization}

The transmit and receive beamformers were both calculated in the same manner in this work, based on the matrix inversion method discussed in [2]. This method required that the desired beamformer without regard to self-interference - often called the quiescent beamformer, denoted $h_{t x, q}$ - first be calculated [6]. Here it was calculated by first calculating the steering vector $\mathbf{c}_{t}$, according to [6]

$$
\mathbf{c}_{t}=e^{j \frac{2 \pi}{\lambda}\left(\mathbf{x}_{t} \sin \left(\theta_{t}\right)+\mathbf{y}_{t} \sin \left(\gamma_{t}\right)\right)},
$$

where $\lambda$ is the wavelength used for antenna spacing, $\mathbf{x}_{t}$ is the $N_{t} \times 1$ vector describing the x-position of all transmit antennas, $\mathbf{y}_{t}$ is the $N_{t} \times 1$ vector describing the y-position of all transmit antennas, and $\theta_{t}$ and $\gamma_{t}$ are the azimuth and elevation angles of the transmit beam, respectively. The corresponding quiescent beamformer was calculated according to [6]

$$
\mathbf{h}_{t, q}=\mathbf{c}_{t}\left(\mathbf{c}_{t}^{H} \mathbf{c}_{t}\right)^{-1}
$$

The final beamformer is calculated as

$$
\mathbf{h}_{t, o p t}=\left(H_{r}^{H} H_{r}+\alpha I_{N_{t}}\right)^{-1} \mathbf{h}_{t, q} \text {, s.t. } \alpha \geq 0,\left\|\mathbf{h}_{T x, O p t}\right\|_{2}=1,
$$

which is a simplified form of the equation presented in [2].

Note that (3) contains the free parameter $\alpha$ used to weight the diagonal of the matrix being inverted. As $\alpha$ increases, the calculated beamformer approaches the quiescent beamformer. 
As $\alpha$ decreases, the beamformer shape is dominated by the goal of minimizing total selfinterference with the receiving antennas. The optimal value for $\alpha$ was sought by optimizing (via MATLAB's fmincon [5]) over the ratio of the beam gain in the direction of transmission to the sum of the gains in the direction of each element in the receive partition. The objective function is (4)

$$
f(\alpha)=\frac{\left|G_{t}\left(\theta_{t}, \gamma_{t}, \alpha\right)\right|}{\sum_{j=1}^{N_{r}}\left|G_{t}(\theta(j), \gamma(j), \alpha)\right|},
$$

where $\theta(j)$ and $\gamma(j)$ are the azimuth and elevation angles, respectively, from the array center to the $j^{\text {th }}$ receive antenna.

Given the optimal $\alpha$ for the transmit beamformer, the optimal beamforming weights $h_{t, o p t}$ are recalculated. The above process is repeated for the receive beamformer, except that all parameters that were with respect to the transmit antennas are now with respect to the receive antennas, and vise versa. In the case of a radar array, the beam main lobe directions are the same for the transmit and receive beams, i.e., $\theta_{t}=\theta_{r}$ and $\gamma_{t}=\gamma_{r}$.

\subsection{Transmit \& Receive Coupling Model}

In order to accurately model the coupling between antennas in the array under study, MITLL provided an S-parameter matrix for a slightly larger array with similar parameters that was then paired down to the correct dimensions for this work. $S$ is the standard S-parameter matrix, described in (5),

$$
S(j, k)=\text { Coupling between Antenna } j \text { and } k
$$

and we define the transmit to receive coupling matrix

$$
H_{t}(j, k)=\text { Coupling between } j^{\text {th }} \text { transmit and } k^{\text {th }} \text { receive antennas }
$$

in (6) and $H_{r}=H_{t}^{T}$. Finally, the interfering signal at the $k^{t h}$ receive antenna at time $t$

$$
s_{\text {int }}(t, k)=H_{t}(:, k)^{T} s_{t}(t,:)
$$

is given in (7).

\subsection{Signal Transmission \& Reflection Model}

Modeling the signal path required an estimate of the signal transmitted by the array of antennas. In order to do so, the gain of each antenna was applied to the input signals, in addition to the complex beamforming weights. Additionally, the noise of each transmit channel $\left(n_{t}(t, j)\right)$ was modeled as independent Additive White Gaussian Noise (AWGN), at $-30 \mathrm{dBm}$. The gain of each antenna was modeled using (8) [7],

$$
G_{\text {ant }}=\frac{4 \pi \eta A_{\text {ant }}}{\lambda^{2}}
$$


where $\eta$ is the efficiency of the antenna, $A_{\text {ant }}$ is the area of the antenna $\left(m^{2}\right)$, and $\lambda$ is the wavelength of antenna operation $(m)$. It should be noted that the value of $\lambda$ used here was actually that used for the center spacing for the array elements, which is nearly equal to the wavelength of the center frequency of the transmitted pulse.

Equation (9) gives the formula for the transmitted signal from the $j^{\text {th }}$ transmit antenna at time $t$ - given the transmit beamformer and the realized antenna gain, considering antenna impedance $Z_{\text {ant }}$.

$$
s_{t x}(t, j)=\mathbf{h}_{t, o p t}(j) \cdot\left(\sqrt{G_{a n t} Z_{a n t}}\left(s_{i}(t)+n_{t}(t, j)\right)\right)
$$

We calculated the far-field approximation of the transmitted signal (along the beam look angle)

$$
s_{f}(t)=\sum_{j=1}^{N_{t}} s_{t}(t, j)
$$

as the coherent sum of the transmitted signal from each antenna, as shown in (10). The signal of interest at each receive antenna can then be modeled as an appropriately scaled and time-shifted version of this far-field signal. The total loss over the transmission path, target reflection, and return path

$$
G_{\text {path }}=\frac{\lambda^{2} A_{\text {target }}}{(4 \pi)^{3} d_{\text {target }}^{4}}
$$

was modeled using the Two-Way Monostatic Radar equation [8, 9], given in (11). $A_{\text {target }}$ is the radar cross section (RCS) of the target and $d_{\text {target }}$ is the distance to the target. This work used a target with $A_{\text {target }}=1 \mathrm{~m}^{2}$ and $d_{\text {target }}=1000 \mathrm{~m}$.

The time delay in the received signal caused by the path length,

$$
t_{\text {path }}=\frac{2 d_{\text {target }}}{c}
$$

was calculated using Equation (12), where $c=3 \cdot 10^{8} \mathrm{~m} / \mathrm{s}$ is the speed of light. Given the gain and delay calculated above, the received signal at antenna $k$ (including the interference signal)

$$
s_{r}(t, k)=\sqrt{G_{\text {path }} G_{\text {ant }} Z_{\text {ant }}} s_{f}\left(t-t_{\text {path }}\right)+s_{\text {int }}(t, k)+n_{r}(t, k)
$$

is given in (13). Again, the model includes independent AWGN receiver noise for each channel $\left(n_{r}(t, k)\right)$, at $-30 \mathrm{dBm}$. 


\subsection{Reference Signal Model}

This work chose a simple model for the reference channel signals for each transmit channel. Each reference channel signal

$$
s_{\text {ref }}(t, j)=s_{t}(t, j)+n_{r e f}(t, j)
$$

is the corresponding transmit channel signal with an additional $-30 \mathrm{dBm}$ independent AWGN noise component, as shown in (14).

\subsection{Self-Interference Cancellation}

There were two options for where to conduct the self-interference cancellation in the signal processing chain. As proposed in [1], an adaptive filter could be developed to form the transmit-channel reference signals into a cancellation signal, which could then be subtracted from the beamformed receive signal. However, initial attempts at this method of cancellation were unsuccessful.

Instead, the reference channel signals $\left(s_{\text {ref }}(t, j)\right)$ were assumed to be good approximations to the transmitted signals, and simply fed through the coupling matrix $\left(H_{t}\right)$ to calculate the cancellation signal

$$
s_{c}(t, k)=H_{t}(:, k)^{T} s_{\text {ref }}(t,:)
$$

for each receive channel. This cancellation method requires two assumptions: that the reference signals are good approximations to the actual transmitted signals and that the coupling matrix is known to high precision. While the first assumption is true in practice, this simulation does not push the limits of this assumption, as the reference signal is simply a noisy version of the transmit signal. The second assumption is also strong - given the ability to characterize the array a priori. The final received, canceled, and beamformed signal

$$
s_{o}(t)=\mathbf{h}_{r, o p t}^{T}\left(s_{t}(t,:)-s_{c}(t,:)\right)
$$

was then calculated as shown in (16).

\subsection{Array Performance Metric \& Optimization}

In order to optimize the partitioning of the given array into transmit and receive elements based on the model above, an objective function that described the performance of a given partition was necessary. The stated goal of this work was to determine if the partitioning of the array could significantly reduce the self-interference between transmit and receive partitions. That being said, a simple measure of the realized self-interference magnitude under these conditions is the Self-Interference Gain $(S I G)$

$$
S I G=10 \log _{10}\left(\mathbf{h}_{t, o p t}^{T} H_{t} \mathbf{h}_{r, o p t}\right)
$$


given in $d B$ by (17). This value captured the ability of the transmit and receive beamformers to work in concert with the array partition - which determines the membership of $H_{t}$ and the dimensions of $\mathbf{h}_{t, o p t}$ and $\mathbf{h}_{r, o p t}$ - to minimize the effects of inter-element coupling present in the received beamformed signal. The model described above was coerced into a function that resulted in the calculation of the objective function given in Equation (17), and MATLAB's genetic algorithm optimization function ga was used to search for the optimal partition [5].

\section{Results \& Discussion}

The results below present an interesting story in light of the stated goals of this work. The array partitions and beam plots are presented below to highlight the nature of each configuration under study. The beamformed received signals (before and after cancellation) and the table presenting the metric for the four different system configurations highlight the relative performance of the configurations.

\subsection{Array Partitions \& Beam Patterns}

Figures 2, 3, and 4 show the partitioning of the array in the cases of the default partition, optimization over just the beams, and optimization over the partition and the beams. The default partition divided the array vertically in half, producing $5 \times 5$ transmit and receive partitions. 


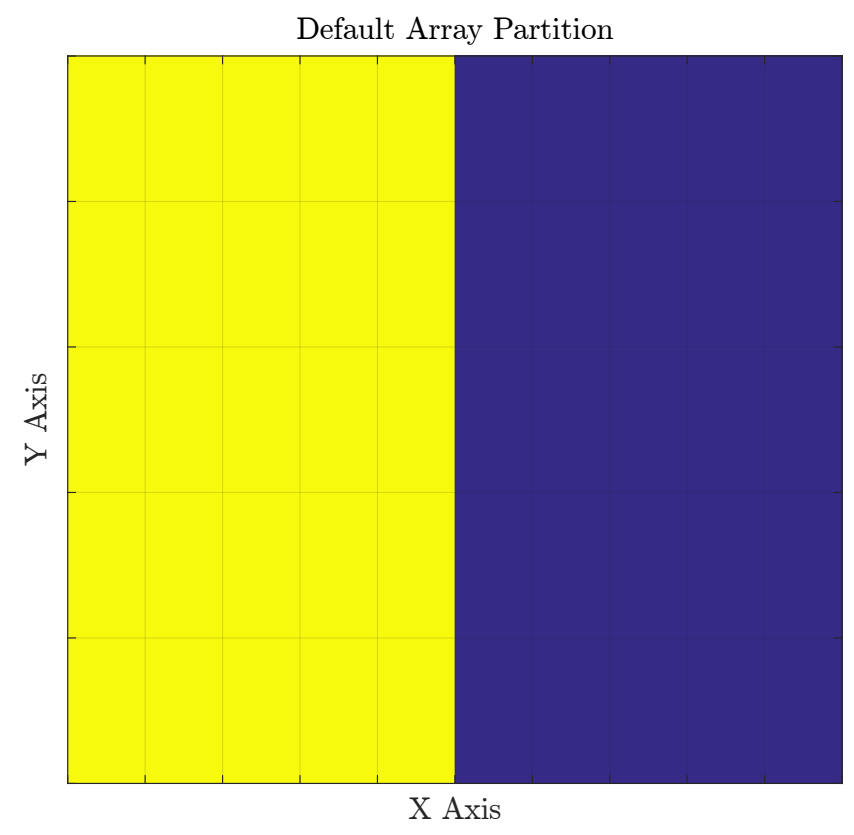

Figure 2: The default array partition studied in $[1,2]$. Yellow $\rightarrow$ Transmit, Blue $\rightarrow$ Receive

Allowing the partition to be optimized, using the quiescent beamformers for both partitions formed a small non-contiguous transmit partition and larger contiguous receive partition, as shown in Figure 3. 


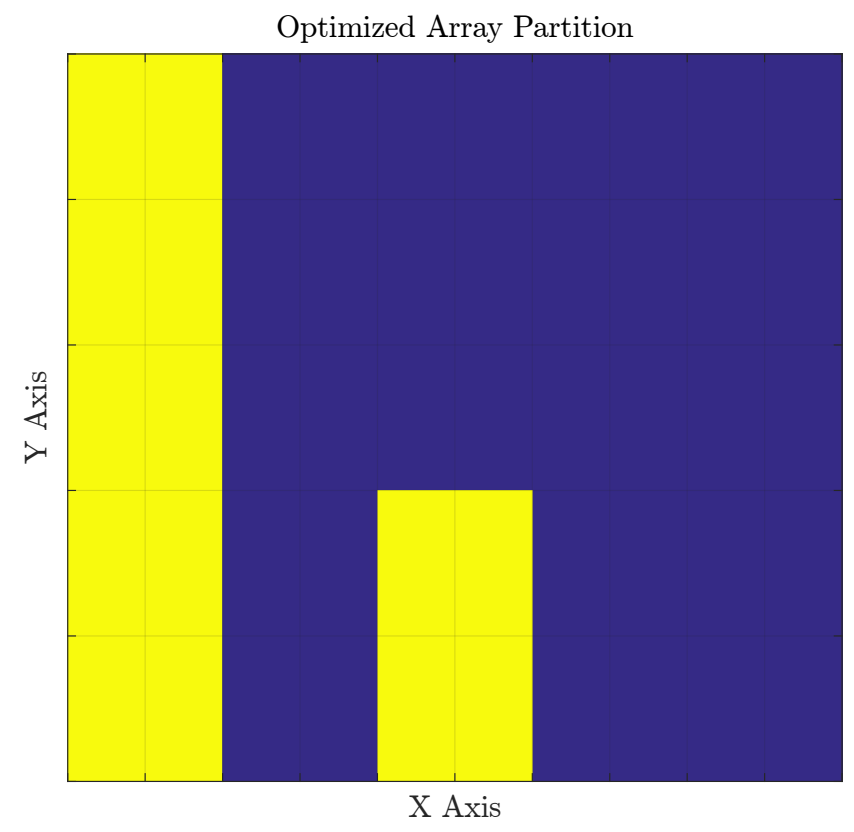

Figure 3: The optimized array partition produced using quiescent beamformers. Yellow $\rightarrow$ Transmit, Blue $\rightarrow$ Receive

Allowing both the partition and the beamformers to be optimized, a non-contiguous highly unconventional partition was produced, as shown in Figure 4. 


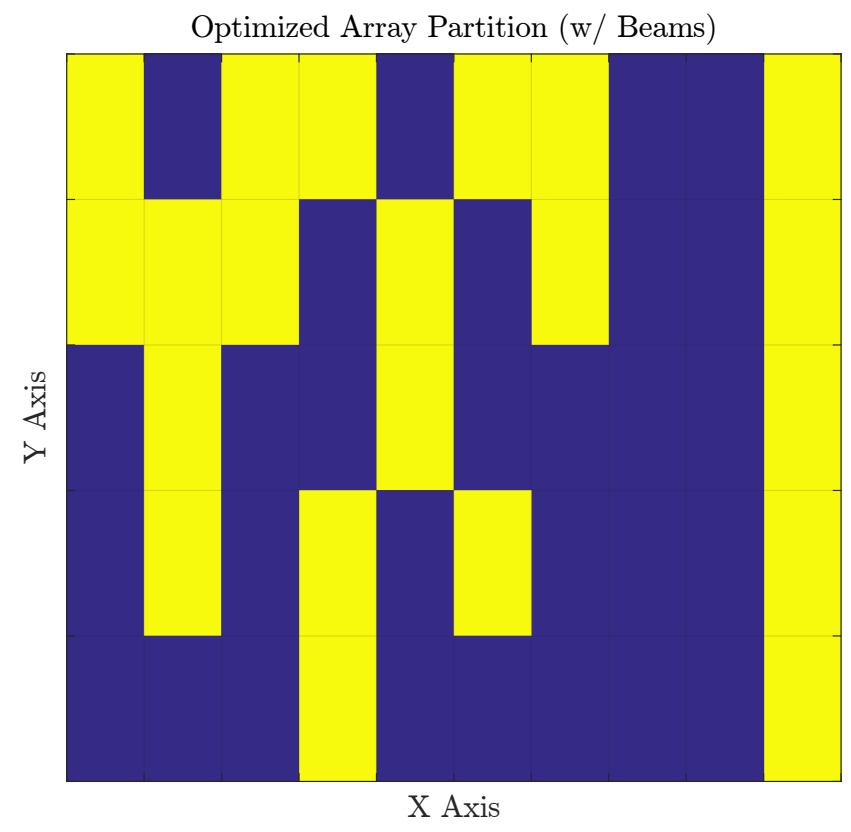

Figure 4: The optimized array partition produced using optimized beamformers. Yellow $\rightarrow$ Transmit, Blue $\rightarrow$ Receive

Figures 5 and 6 show the quiescent transmit and receive beams (respectively) formed for the default partitions.

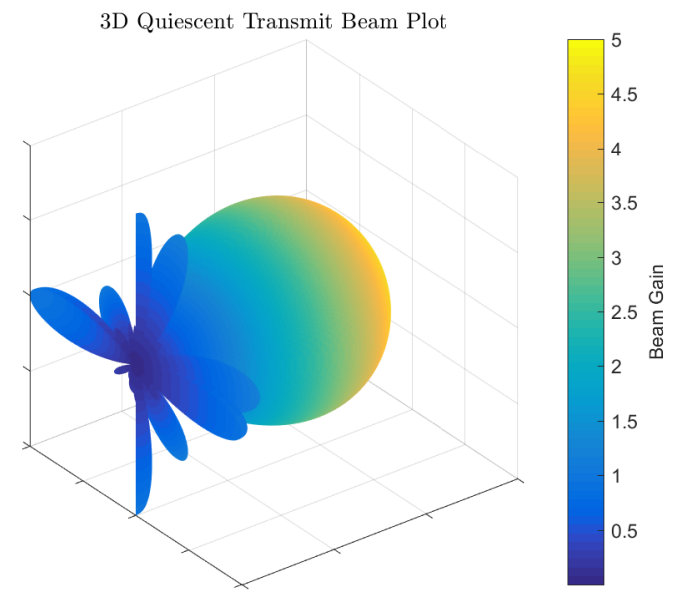

Figure 5: The quiescent transmit beam produced from the default array partition

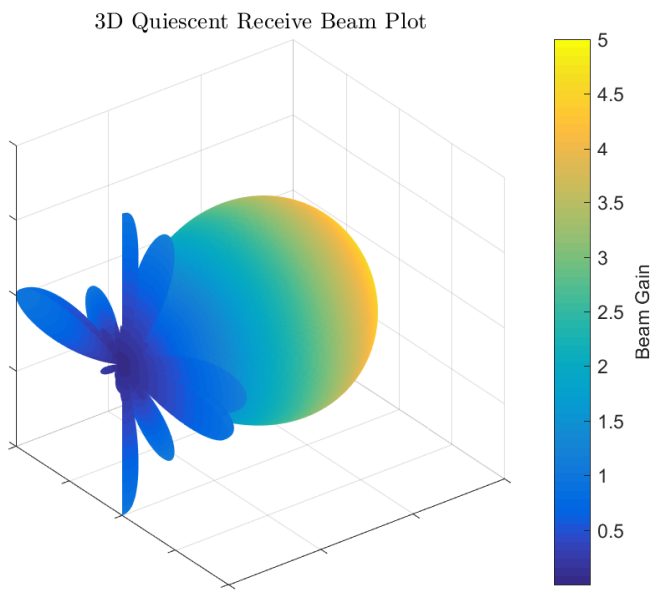

Figure 6: The quiescent receive beam produced from the default array partition

Figures 7 and 8 show the optimized transmit and receive beams for the default partition. 


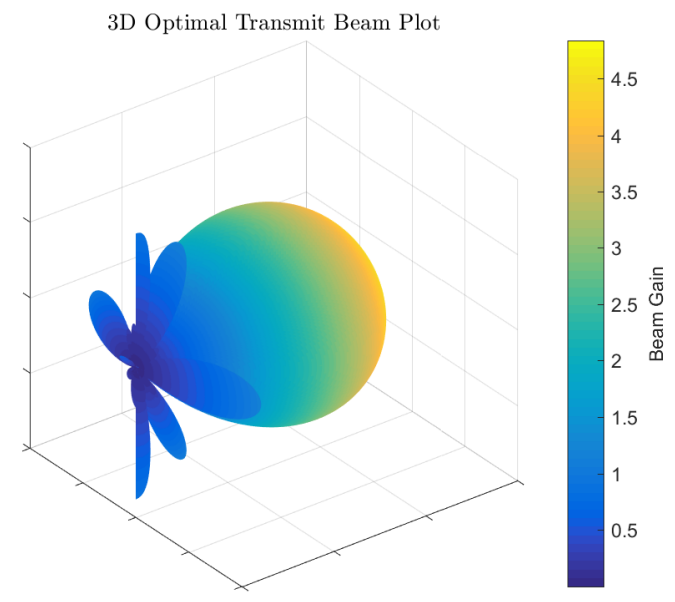

Figure 7: The optimized transmit beam produced from the default array partition

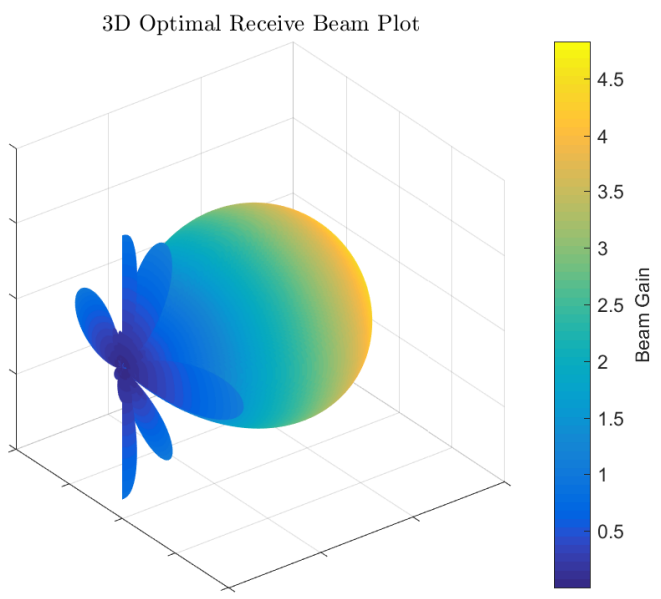

Figure 8: The optimized receive beam produced from the default array partition

Figures 9 and 10 show the beams resulting from optimizing the partition, but sticking to quiescent beams.

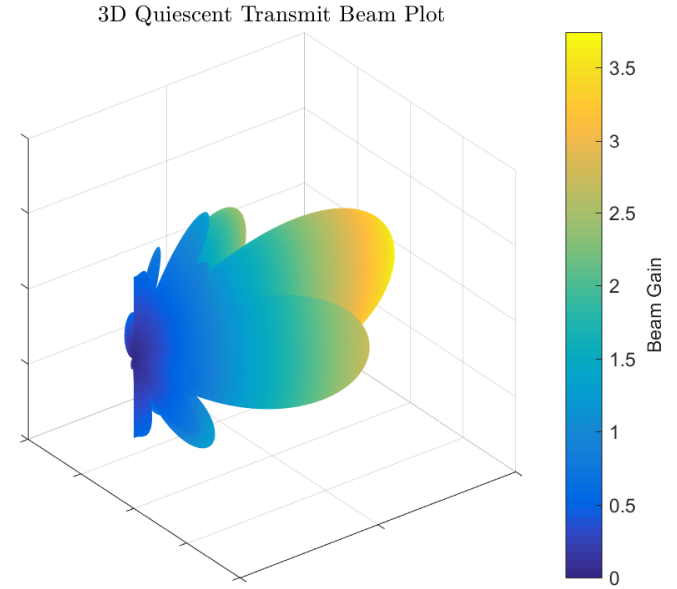

Figure 9: The quiescent transmit beam produced from the optimized array partition

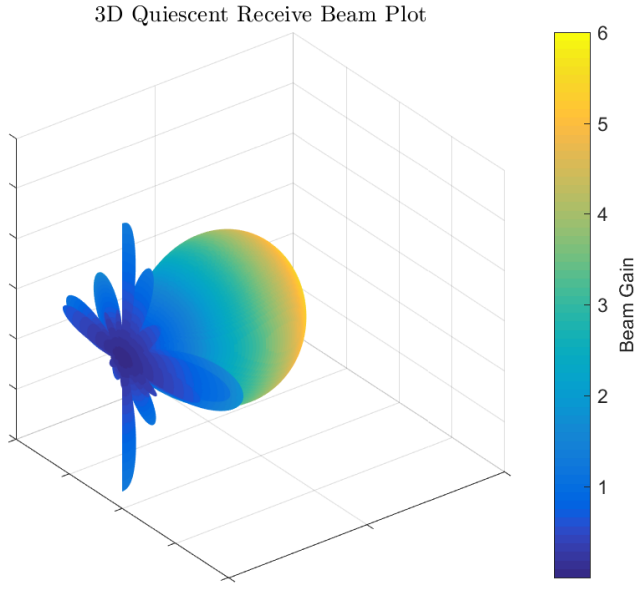

Figure 10: The quiescent receive beam produced from the optimized array partition

Figures 11 and 12 show the beams produced when the partition and beams were optimized together. 


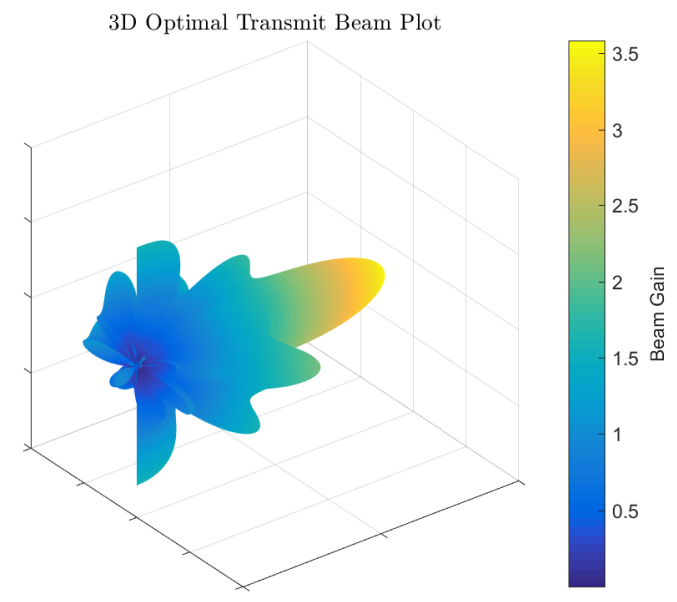

Figure 11: The optimized transmit beam produced from the optimized array partition

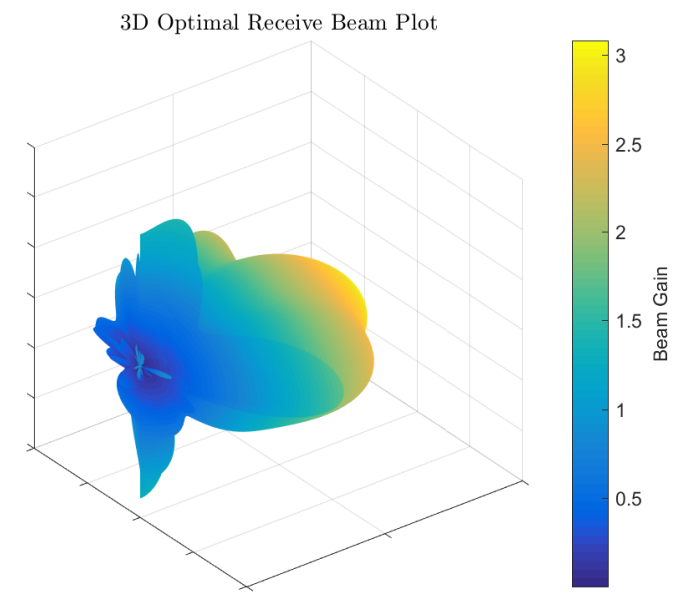

Figure 12: The optimized receive beam produced from the optimized array partition

The beams produced in both cases where the partition was optimized display irregular sidelobe structures, when compared with the beams from the default partition. It is difficult to compare the beams visually to determine their performance in terms of minimizing selfinterference.

\subsection{Array Performance Metric Summary}

The easiest comparison between configurations involves looking at the metric in Table 1.

\begin{tabular}{ccc} 
Partition & Beams & SIG $(d B)$ \\
\hline Default & Quiescent & -19.9 \\
Default & Optimized & -30.4 \\
Optimized & Quiescent & -17.0 \\
Optimized & Optimized & -108.3 \\
\hline
\end{tabular}

Table 1: Self-Interference Gain $(d B)$ for each combination of optimizations.

In this case, a lower value of Self-Interference Gain is preferable because that indicates a reduced level of interference in the received signal. According to the table, the default partition with optimized beams demonstrated some performance gain over the default partition and default beams. It also appears that allowing the partition and beams to be optimized simultaneously significantly increases the potential for performance increase, while optimizing the partitioning alone was actually detrimental. That is concerning, because the optimized partition should at least achieve the performance of the default partition and quiescent beams. 
This suggests that the objective functions used and the setup of the optimization algorithm both bear further study.

\subsection{Realized Self-Interference Magnitude}

Figures 13 and 14 show the beamformed signal before and after cancellation (respectively) for the default partition with quiescent beams.
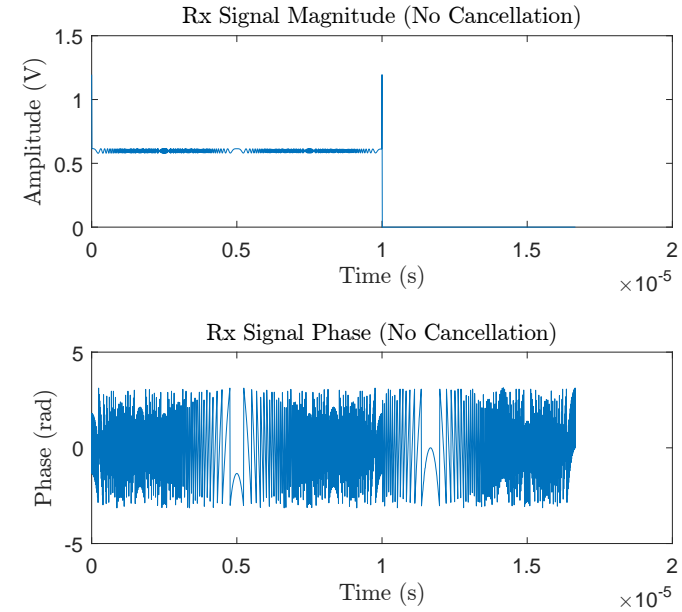

Figure 13: The beamformed signal before self-interference cancellation, using the quiescent beams produced from the default array partition
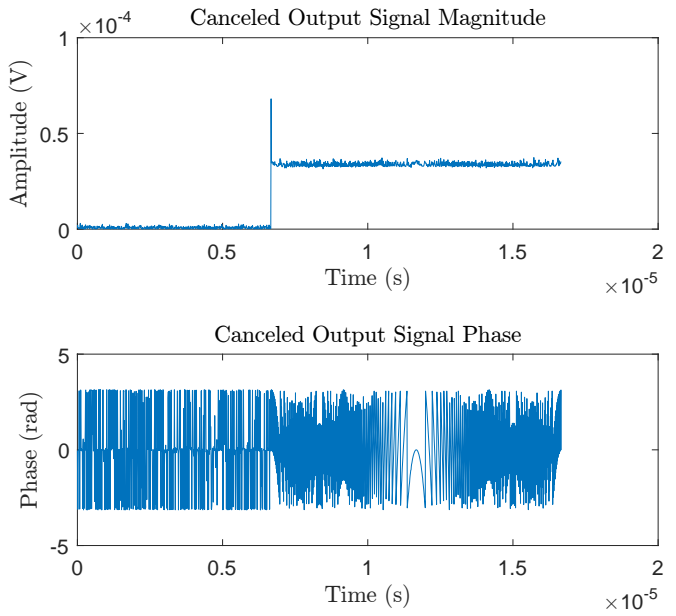

Figure 14: The beamformed signal after self-interference cancellation, using the quiescent beams produced from the default array partition

The signal of interest is clearly visible in Figure 14 , beginning at $t \approx 0.7 \cdot 10^{-5} \mathrm{~s}$, while it is completely drowned out in Figure 13 by the self-interference component that begins at $t=0 \mathrm{~s}$. Figures 15 and 16 show the same signals for the case where the transmit and receive beams were optimized over the default array partition. 

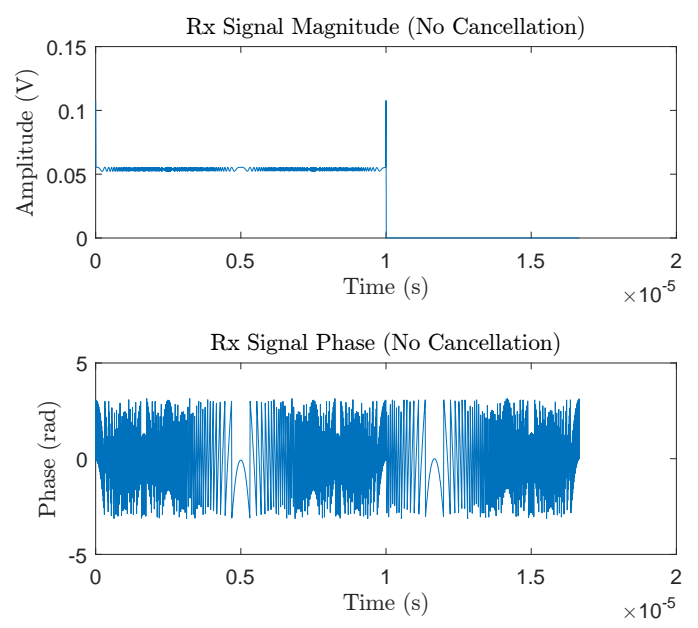

Figure 15: The beamformed signal before self-interference cancellation, using the optimized beams produced from the default array partition
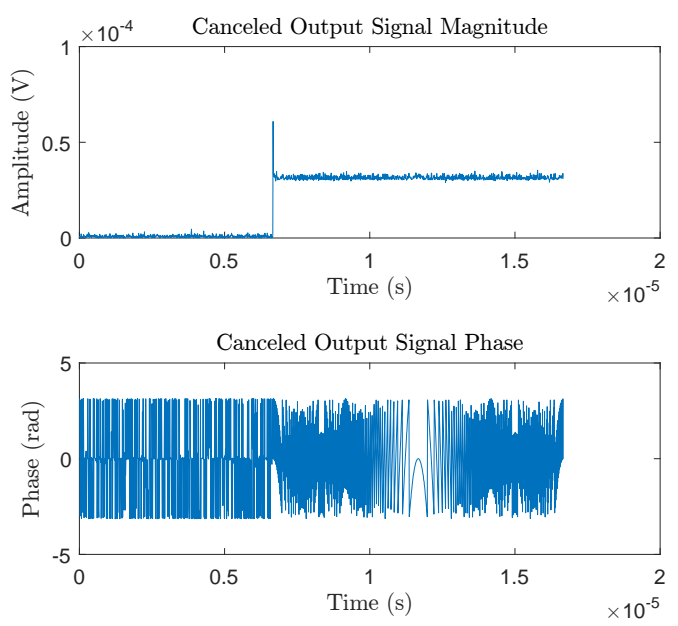

Figure 16: The beamformed signal after self-interference cancellation, using the optimized beams produced from the default array partition

Here it should be noted that the self-interference component (Figure 15) is significantly reduced compared to the case where the default partition and quiescent beams were employed. Additionally, the magnitude of the signal of interest (Figure 16) is approximately the same magnitude as the default case. Figures 17 and 18 show the beamformed signals with out and with self-interference cancellation, in the case where the partition was optimized using quiescent beamformers.
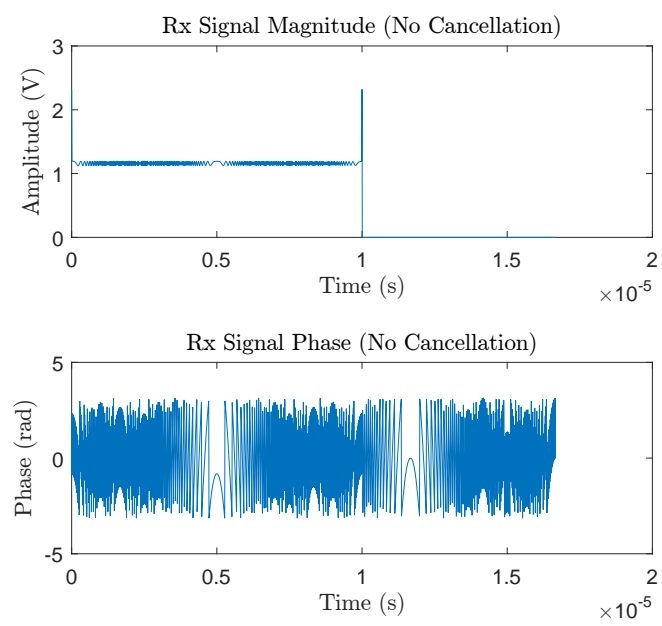

Figure 17: The beamformed signal before self-interference cancellation, using the quiescent beams produced from the optimized array partition
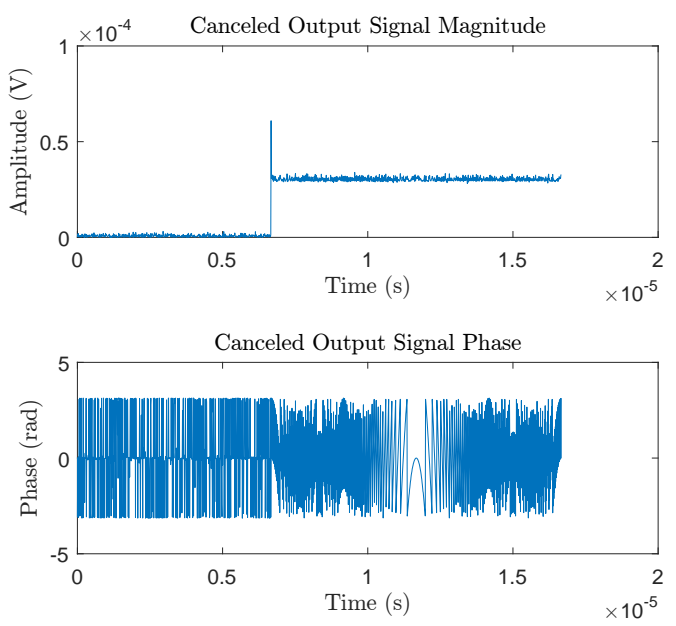

Figure 18: The beamformed signal after self-interference cancellation, using the quiescent beams produced from the optimized array partition 
These figures show a level of self-interference that is about twice that of the default partition, quiescent beams case, while the signal of interest magnitude is approximately the same as the default partition, quiescent beams case. Figures 19 and 20 show the beamformed received signals without and with self-interference cancellation (respectively) for the optimized partition, optimized beams case.
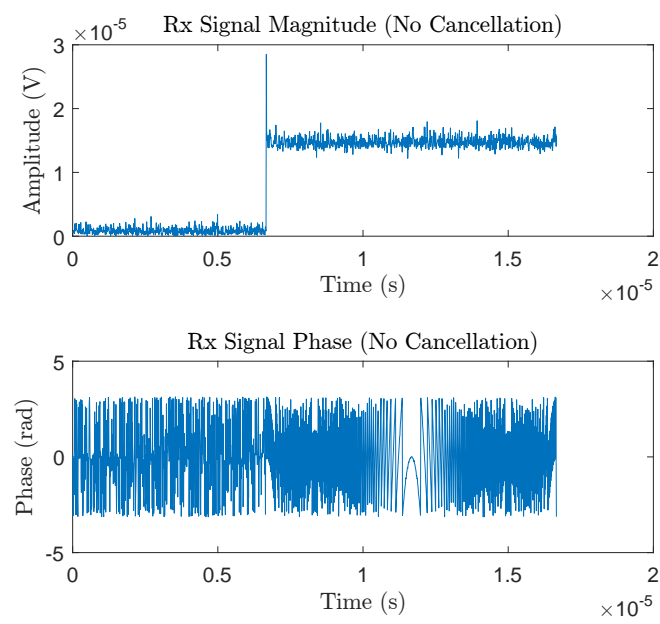

Figure 19: The beamformed signal before self-interference cancellation, using the optimized beams produced from the optimized array partition
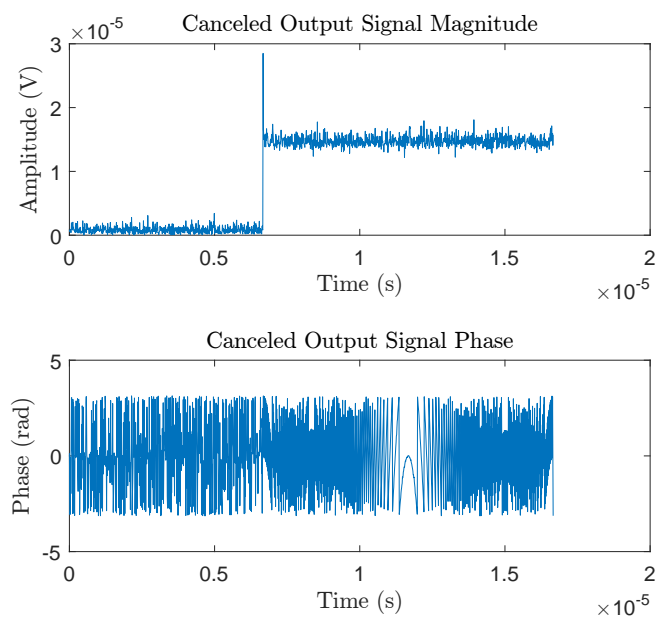

Figure 20: The beamformed signal after self-interference cancellation, using the optimized beams produced from the optimized array partition

These plots demonstrate that the optimization algorithm was able to reduce the self-interference magnitude enough to make the signal of interest clearly visible in the receive beamformed signal without cancellation. However, the signal of interest magnitude was about half of that found in the other cases.

It should be noted that the results of the partition optimization were not stable from run to run. In other words, a different partition was returned as optimal after each run, suggesting that the optimization space may contain a large number of local optima. Furthermore, in the cases where the partition was optimized, the graphs and performance metric were produced by feeding the optimal partition back into the objective function. In the case where both the partition and beams were optimized, this implies that the beams were recalculated, but the optimization algorithm for the beams given a partition appeared stable.

\section{Conclusions}

Given the data presented above, the goal to develop a model suitable for array partition optimization was met. Additionally, this work demonstrates that the realized self-interference 
can be significantly affected by the partitioning of the array. However, additional research into the correct objective functions and optimization routines is necessary. Additionally, simply minimizing the predicted level of self-interference may not be a sufficient metric for array performance optimization, as it does not guarantee that individual receive antennas will not become saturated. Further study into the nature of the optimization space will be required to solve this complex problem.

\subsection{Future Work}

This work opens opportunities for a great deal of additional work. While this work simulated the array in a radar mode, and $[1,2]$ simulated the array in a communications mode, electronic warfare would be a natural third mode of operation to explore [3]. In fact, once all three modes have been explored individually, it would make sense to consider the necessary algorithms to allow multiple modes operating simultaneously on one array.

Looking closely at the scope of this work, there are three main areas that could be expanded upon, including: transmit and receive beamformer optimization, robust self-interference cancellation, and array partitioning intuition and methods.

Regarding the beamformers used in this work, several improvements could be made. First, it should be determined whether or not a closed form solution for $\alpha$ exists. Second, the objective function should be updated to include inter-element coupling effects. Third, the possibility of simultaneously optimizing the transmit and receive beamformers to increase overall isolation should be explored. Finally, other beamformers should be considered that may be less computationally expensive or may offer better performance under more realistic conditions.

In determining the robustness of the current self-interference cancellation approach, the first step would be to perturb the coupling matrix used to calculate the cancellation signals in relation to the coupling matrix used to calculate the interference signals and test the performance of the simplistic cancellation system. Should the current simplistic method not provide the desired performance, a more robust method should be pursued.

Finally, further investigation into the mechanisms that create optimal arrays should be pursued, as the use of the genetic algorithm for optimization is computationally expensive and impractical. One potential method for array partitioning would be a greedy approach where elements would be added to the transmit partition in such a way that they reduce or add the minimum possible amount to the realized self-interference. 


\section{References}

[1] Jonathan P. Doane, Kenneth E. Kolodziej, and Bradley T. Perry. Simultaneous transmit and receive with digital phased arrays. In Proc. 2016 IEEE International Symposium on Phased Array Systems and Technology, Waltham, MA, USA, Oct 18-21 2016.

[2] Jonathan P. Doane, Kenneth E. Kolodziej, and Bradley T. Perry. Simultaneous transmit and receive with digital phased arrays. Unpublished extended version of paper of same title appearing in Proc. 2016 IEEE International Symposium on Phased Array Systems and Technology, 2016.

[3] Jonathan P. Doane. Phone discussion regarding star work progress, 7 Feb 2017.

[4] V.V. Veeravalli. Complex baseband representation. ECE 461 Course Notes, 31 Aug 2006.

[5] The MathWorks, Inc. MATLAB Help Documentation.

[6] Simon Haykin. Adaptive Filter Theory. Pearson, fifth edition, 2014.

[7] Kirt Blattenberger. Antenna introduction / basics, 2016.

[8] Kirt Blattenberger. Two-way radar equation (monostatic), 2016.

[9] Kirt Blattenberger. Radar cross section (rcs), 2016. 